\title{
Plasmodium Vivax Malaria Presenting With Severe Thrombocytopenia
}

\author{
Ravinder Pal Singh Makkar, Surabhi Mukhopadhyay \\ Amitabh Monga and Ajay Kr. Gupta
}

\author{
Department of Internal Medicine, Sitaram \\ Bhartia Institute of Science ans Research, New \\ Delhi, India
}

\begin{abstract}
Plasmodium falciparum and Plasmodium vivax malaria are endemic infections in India and are commonly associated with mild hematological abnormalities. Severe thrombocytopenia is common in isolated falciparum and mixed falciparum/vivax malaria, but is very rare in isolated P.vivax infection. We hereby report a case of severe thrombocytopenia (platelet count of $8 \times 10^{9} / \mathrm{L}$ ) in a case of vivax malaria. This is only the second case ever reported in the literature of such profound thrombocytopenia in a case of isolated P.vivax malaria.
\end{abstract}

Key Words: Plasmodium vivax, malaria, severe thrombocytopenia.

Malaria is common in most parts of India and is usually caused by $P$. falciparum and $P$. vivax. Though mild thrombocytopenia is common in both falciparum and vivax malaria, severe thrombocytopenia is reported especially in $P$. falciparum malaria and is very rare in isolated $P$. vivax infection. Both non-immunological as well as immunological destruction of platelets have been implicated in causing thrombocytopenia in such cases but the mechanisms involved are still not completely clear.

\section{Case Report}

A 43-year-old male, resident of eastern India, arrived at the hospital emergency room with complaints of high grade, intermittent fever associated with chills and rigors during seven days. He also had a two-day history of mild spontaneous bleeding from the gums. There was no history of bleeding from any other site

Received on 12 June 2002; revised 04 October 2002.

Address for correspondence: Dr. Ravinder Pal Singh Makkar, MD, Attending Physician, Department of Internal Medicine, Sitaram Bhartia Institute of Science and Research, B-16, Mehrauli Institutional Area, New Delhi-110016, India.

E-mail-makkar_r@yahoo.com

The Brazilian Journal of Infectious Diseases 2002;6(5):263-265 (C) 2002 by The Brazilian Journal of Infectious Diseases and Contexto Publishing. All rights reserved.

$1413-8670$ and no past or family history suggestive of bleeding diathesis. There was no history of any drug ingestion. On examination, the patient was febrile with a temperature of $40^{\circ} \mathrm{C}$. He had slight pallor and the liver and spleen were palpable $2 \mathrm{~cm}$ and $3 \mathrm{~cm}$ below the costal margin, respectively. There were no purpuric spots and the tourniquet test was negative.

Routine blood investigations revealed $7.5 \mathrm{~g} / \mathrm{dL}$ hemoglobin and a WBC count of $6.3 \times 10^{9} / \mathrm{L}$, with $54 \%$ neutrophils, 39\% lymphocytes, $4 \%$ eosinophils and 3\% monocytes. The platelet count was $8 \times 10^{9} / \mathrm{L}$. The peripheral blood smear showed numerous gametocytes of $P$. vivax in a background of marked thrombocytopenia. The 'OptiMAL® Rapid Malaria Dipstick Test' (DiaMed AG,Switzerland) for $P$. falciparum was negative but was positive for $P$. vivax. The bleeding time was prolonged to 9 minutes while the clotting time was 3 minutes, with a prothrombin time of 14 seconds (control 13 seconds) and activated partial thromboplastin time of 40 seconds (control 36 seconds). Glucose-6-phosphate dehydrogenase levels were normal and disseminated intravascular coagulation (DIC) was ruled out by normal levels of fibrinogen degradation products (FDP). Total serum bilirubin was $1.4 \mathrm{mg} / \mathrm{dL}$ with normal serum transaminase levels. Dengue serology (IgG and IgM) and Elisa for HIV were nonreactive. The patient was treated with antimalarials (quinine sulphate $10 \mathrm{mg} / \mathrm{kg}$ thrice daily) for 7 days and was given 6 units of platelet transfusions 
during the course of the hospital stay. He improved clinically and became afebrile on the third day of hospital stay. The peripheral blood smear showed clearance of parasitemia from the third day and became negative for malarial parasites on the fifth day. The platelet count increased from $8 \times 10^{9} / \mathrm{L}$ on admission to $340 \times 10^{9} / \mathrm{L}$ at the time of discharge. The hemoglobin also improved to $10.5 \mathrm{~g} / \mathrm{dL}$. There was no bleeding from any site at any time during the hospital stay and he was discharged on the 7 th day in a stable condition.

\section{Discussion}

Malaria is a common infection in most parts of India and is commonly associated with mild thrombocytopenia [1]. Profound thrombocytopenia is a well-recognized complication of falciparum malaria but has been less well described in vivax malaria. Of 173 cases of malaria in U.S. soldiers reported by Martelo et al [2] in 1969, 93\% had $P$. vivax but only $15 \%$ had thrombocytopenia with no documentation of the lowest platelet count. In Horstmann's series [3], the lowest count in 39 cases of vivax malaria was 44x10\%/L. Pukrittayakamee et al. [4] described a case of a volunteer experimentally infected with the Chesson's strain of $P$. vivax with a platelet count of $20 \times 10^{9} / \mathrm{L}$. Recently a case of vivax malaria associated with an initial platelet count of $5 \times 10^{9} / \mathrm{L}$ was reported from India [5]. In our case, the patient presented with a severe thrombocytopenia of $8 \times 10^{9} / \mathrm{L}$, with spontaneous bleeding from the gums. This is probably only the second case ever reported of isolated $P$. vivax infection causing such profound thrombocytopenia.

The mechanism of thrombocytopenia in malaria is not clearly known. Fajardo and Tallent [6] in 1974 demonstrated $P$. vivax within platelets by electron microscopy and suggested a direct lytic effect of the parasite on the platelets. Both non-immunological destruction [7] as well as immune mechanisms involving specific platelet-associated IgG antibodies that bind directly to the malarial antigen in the platelets have been recently reported to play a role in the lysis of platelets and the development of thrombocytopenia [8]. In clinical trials, recombinant - macrophage colony stimulating factor (M-CSF) has been known to cause a reversible dose dependent thrombocytopenia. Elevated M-CSF levels in malaria, by increasing macrophage activity may mediate platelet destruction in such cases [9]. Oxidative stress damage of thrombocytes has also been implicated in the etiopathogenesis based on the finding of low levels of platelet superoxide-dismutase and glutathioneperoxidase activity and high platelet lipid peroxidation levels in malaria patients, when compared to those of healthy subjects [10].

Since such severe thrombocytopenia is rare in vivax malaria, and mixed infection with falciparum and vivax is common in India, it could be argued that this case is associated with coexistent $P$. falciparum infection. To rule out concomitant $P$. falciparum infection, an 'OptiMAL® Rapid Malaria Dipstick Test' was done, which was negative. This test detects the presence of Plasmodium lactate dehydrogenase (pLDH); an enzyme produced both by the sexual and asexual forms of the parasite. The presence of $\mathrm{pLDH}$ is revealed using monoclonal antibodies directed against isoforms of the enzyme. This test detects parasitemia levels of 100-200 parasites per $\mu \mathrm{L}$ of blood (corresponding to a parasitemia of $0.002 \%-0.004$ $\%)$. Another point against the presence of associated falciparum infection is that such a low parasitemia of P.falciparum so as to be undetectable both by the dipstick test and standard microscopy, is highly unlikely to cause such profound thrombocytopenia. This case therefore highlights the fact that in patients with acute febrile illness and with marked thrombocytopenia, P.vivax should also be kept as a differential diagnosis and treated according to the local drug resistance pattern prevalent in the area.

\section{References}

1. Looareesuwan S., Davis J.G., Allen D.L., et al. Thrombocytopenia in malaria. Southeast Asian J Trop Med Public Health 1992;23:44.

2. Martelo O.J., Smoller M., Saladin T.A. Malaria in American soldiers. Arch Int Med 1969; 123:383-7.

3. Horstmann R.D., Dietrich M., Bienzle U., Rasche H. Malaria induced thrombocytopenia. Blood 1991;42:157-64. 
4. Pukrittayakamee S., White N.J., Clemens R., et al.Activation of the coagulation cascade in falciparum malaria. Trans R Soc Trop Med Hyg 1989; 83:762-6.

5. Kakar A., Bhoi S., Prakash V., Kakar S. Profound thrombocytopenia in Plasmodium vivax malaria. Diagn Microbiol Infect Dis 1999;35:243-4.

6. Fajardo L.F., Tallent C. Malarial parasites within human platelets. JAMA 1974;229:1205.

7. Looaresuwan S., Davis J.G., Allen D.L., et al. Thrombocytopenia in malaria. Southeast Asian J Trop Med Public Health 1992;23(1):44-50.

8. Yamaguchi S., Kubota T., Yamagishi T., et al. Severe thrombocytopenia suggesting immunological mechanism in two cases of vivax malaria. Am J Hematol 1997;56(3):183-6.

9. Lee S.H., Looaresuwan S., Chan J., et al. Plasma macrophage colony stimulating factor and P-selectin levels in malaria associated thrombocytopenia. Thromb Haemost 1997;77(2):289-93.

10. Erel O., Vural H., Aksoy N., et al. Oxidative stress of platelets and thrombocytopenia in patients with vivax malaria. Clin Biochem 2001;34(4):341-4. 